\title{
Presencias y ausencias sobre género y feminismos en publicaciones de Trabajo Social
}

\author{
Presences and absences on gender and feminisms in social \\ work publications
}

Presenças e ausências sobre gênero e feminismos em publicações de Serviço Social

Cory Duarte Hidalgo* , Viviana Rodríguez Venegas**

\section{RESUMEN}

Revisión sistematizada que indaga en artículos publicados entre 2014 y 2019, respecto de la relación entre género, feminismos y Trabajo Social, en revistas de especialidad en Chile, Argentina, Colombia, Puerto Rico, Brasil y España. Los resultados demuestran acercamientos teórico-conceptuales, metodológicos y principales discusiones que desde el Trabajo Social se exponen para establecer las presencias y ausencias de género y feminismos en la literatura especializada de la disciplina. Los resultados permiten seguir las pistas de un Trabajo Social feminista agenciado desde los márgenes, cuyas tramas concluyentes se sitúan en la responsabilidad ética-política acuerpada, la formación en metodologías críticas feministas, la trascendencia de la perspectiva de género y el (re)posicionamiento del quehacer disciplinar en/con actoras/es y movimientos sociales en pluralidad y diversidad de luchas y resistencias.
Palabras clave: feminismos, género, Trabajo Social, Trabajo Social feminista.

\footnotetext{
* Chilena, doctora en Trabajo Social. Académica Departamento de Trabajo Social de la Facultad de Ciencias Jurídicas y Sociales, Universidad de Atacama. E-mail: cory.duarte@uda.cl

** Chilena, doctoranda en Estudios Sociales de América Latina. Académica Departamento de Trabajo Social de la Facultad de Ciencias Jurídicas y Sociales, Universidad de Atacama. E-mail: viviana.rodriguez@uda.cl
} 


\begin{abstract}
This article is a systematized review investigating articles published between 2014 and 2019 regarding the relationship between gender, feminism, and Social Work, in specialized journals in Chile, Argentina, Colombia, Puerto Rico, Brazil, and Spain. The results show theoretical-conceptual and methodological approaches and the main discussions that Social Work presents to establish the presence and absence of gender and feminism in the discipline's specialized literature. The results allow us to follow the tracks of a feminist Social Work managed from the margins, whose conclusive plots are located in the agreed ethical-political responsibility, the training in critical feminist methodologies, the transcendence of the gender perspective, and the (re)positioning of the disciplinary work in/with actors and social movements in plurality and diversity of struggles and resistances.
\end{abstract}

\section{RESUMO}

Revisão sistematizada que investiga artigos publicados entre 2014 e 2019, sobre a relação entre gênero, feminismo e serviço social, em revistas especializadas no Chile, Argentina, Colômbia, Porto Rico, Brasil e Espanha. Os resultados apresentam abordagens teórico-conceituais e metodológicas e principais discussões que se expõem a partir do serviço social para estabelecer as presenças e ausências de gênero e feminismo na literatura especializada da disciplina. Os resultados nos permitem seguir os rastros de uma agência feminista de assistência social à margem, cujos enredos conclusivos se situam na responsabilidade ético-política instituída, na formação em metodologias feministas críticas, na transcendência da perspectiva de gênero e no (re) posicionamento do trabalho disciplinar em / com atores e movimentos sociais na pluralidade e diversidade de lutas e resistências.

\section{Key}

words: feminisms, gender, Social Work, feminist social work.
Palavras-chave: feminismos, gênero, serviço social, serviço social feminista. 


\section{Presentación}

Cuando en las calles de nuestro país se organiza una movilización social sin precedentes, conformando un cuerpo político, plural y obstinado (Butler, 2017), en el que emergen imaginarios políticos marginados (Muñoz, 2020), cabe la pregunta respecto de las formas en que las trabajadoras sociales ${ }^{1}$ nos relacionamos con las comunidades y movimientos sociales, introduciendo en la reflexión y en la acción, categorías y conceptos provenientes desde los feminismos y otras disidencias al modelo hegemónico.

El estallido social de octubre de 2019 en Chile y las continuas crisis obligan a replantear la formación de profesionales de la intervención social, revisando, cuestionando y repensando las interacciones, cercanas y distantes, que sostiene la academia, no solo con quienes están en la primera línea de la intervención en lo social, sino también con aquellos y aquellas que están en la calle articulando acciones de resistencia activa. Así, el presente trabajo intenta proponer una discusión sobre algunos elementos actualmente presentes en las discusiones feministas, pero que se manifiestan ausentes en la formación profesional, de corte generalista y aséptica (Duarte, 2013), que creemos relevantes de considerar y problematizar acorde al contexto actual de nuestro país y la riqueza de sus acontecimientos.

Se plantean algunos ejes de ausencia que han sido escasamente tratados en Trabajo Social, pero que emergen como presencias a través de las reflexiones y acciones feministas, y que, a la vez, intentan alejarse de los lugares comunes que adopta la reflexión disciplinar cuando problematiza elementos asociados al género, asumiendo identidades profesionales basadas en la composición y corporalidad femenina de sus actuantes.

El artículo inicia con la presentación del método escogido en la realización del estudio, para luego exponer resultados generales y detenerse en una discusión sobre el tema tratado, finalizando con conclusiones que abordan los objetivos trazados en esta investigación.

1 Quienes suscriben el artículo han tomado la decisión política de escribir utilizando solo el género femenino, como forma de resistencia y visibilización de las mujeres que mayoritariamente ejercemos como trabajadoras sociales. 


\section{Método}

La revisión de la literatura respecto del tema de este artículo, consideró aquella publicada entre 2014 y 2019 en revistas de Trabajo Social ingresadas en el índice ERIH Plus (European Reference Index for the Humanities and Social Sciences), por ser esta una indexación reconocida por la Agencia Nacional de Investigación y Desarrollo (ANID) en la evaluación de proyectos de investigación la que, además, resulta ser un índice común a varias revistas de Trabajo Social a nivel iberoamericano.

La revisión sistemática es una metodología utilizada en distintas disciplinas, destacando su uso en las publicaciones del área de salud, la que exige "un método riguroso y explícito para la identificación, evaluación crítica y síntesis de la evidencia obtenida" (Beltrán, 2005, p. 61). En este sentido, este tipo de estudios, a partir del uso de una metodología cualitativa, permite ampliar la comprensión de los fenómenos (Grant y Booth, 2009).

Las revisiones sistemáticas incluyen un proceso de revisión basado en fases críticas asociadas a conceptos centrales que dirigen la búsqueda. María Grant y Andrew Booth, en 2009, acuñan el memotécnico SALSA, que en inglés hace referencia a "Search, AppraisaL, Synthesis and Analysis", es decir un proceso crítico que implica la Búsqueda, Evaluación, Síntesis y Análisis del material bibliográfico. No obstante, en ciencias sociales las revisiones bibliográficas tienen el carácter de sistematizadas, para explorar elementos asociados a distintos ámbitos de investigación, identificando "las tendencias y corrientes principales en un área, así como la detección de huecos y oportunidades de investigación" (Codina, 2018, p. 11), lo que permite, como en este caso, realizar un "estado del arte" del tema en cuestión.

En este estudio se definió como objetivo central el indagar en los artículos publicados entre 2014 y 2019, respecto de la relación entre género, feminismos y Trabajo Social, en revistas de especialidad en Chile, Argentina, Colombia, Puerto Rico, Brasil y España. A su vez, se intentó identificar los acercamientos conceptuales a la noción de "género", reconocer las temáticas que desde el Trabajo Social se investigan con relación a géneros y feminismos, y establecer las presencias y ausencias en la literatura especializada de Trabajo Social respecto del tema aquí tratado.

Al optar por realizar una revisión sistematizada de la literatura, se definió una estrategia de búsqueda para la identificación de estudios a 
considerar en la investigación. La búsqueda tuvo por propósito, en una primera instancia, identificar los trabajos publicados entre 2014 y 2019 sobre la relación entre género, feminismos y Trabajo Social, en revistas indexadas en ERIH PLUS. La identificación de estudios se realizó mediante una búsqueda en las páginas web de 10 revistas de Trabajo Social previamente seleccionadas. En la búsqueda se incluyó una serie de términos o palabras claves que permitieron guiar la indagación propuesta, estableciéndose como claves los conceptos de "género", "feminismo", "sexualidad" y "mujeres".

Los registros identificados en la búsqueda se introdujeron de forma secuencial en una base de datos creada por las investigadoras, identificando y eliminando los ingresos duplicados. Posteriormente se trabajó siguiendo una serie de criterios de inclusión y exclusión, establecidos con anterioridad, lo que facilitó seleccionar el material obtenido en la primera búsqueda. Los criterios de inclusión de los artículos tenían relación con la especificidad de estos, estableciendo para ello algunas directrices señaladas en la tabla 1:

Tabla 1.

Criterios de inclusión para el proceso de selección

\begin{tabular}{ll}
\hline Tipo de artículos & $\begin{array}{l}\text { Artículos de discusión teórica, investigaciones } \\
\text { sociales o sistematizaciones. }\end{array}$ \\
\hline Tipo de revistas & $\begin{array}{l}\text { Revistas de Trabajo Social. } \\
\text { Indexadas en ERIH PLUS }\end{array}$ \\
& $\begin{array}{l}\text { Acceso abierto. } \\
\text { Que presenten una continuidad en la publicación a } \\
\text { partir del año 2014. }\end{array}$ \\
\hline Criterio temporal & $\begin{array}{l}\text { Artículos publicados entre el 1 de enero de 2014 y el } \\
\text { 31 de diciembre de 2019. }\end{array}$ \\
\hline Criterio lingüístico & Estudios publicados en idioma español. \\
\hline Palabras claves & $\begin{array}{l}\text { Considera en el título o en la selección de conceptos } \\
\text { claves, al menos una de las siguientes palabras: } \\
\text { género, feminismo, sexual, mujer/mujeres. }\end{array}$ \\
\hline
\end{tabular}

Fuente: Elaboración propia.

2 En una primera búsqueda se identificaron 10 revistas indexadas en ERIH PLUS, sin embargo, se excluyeron tres que no tenían una continuidad en la publicación, según lo dispuesto como criterio de inclusión. Finalmente, las revistas consideradas para este estudio fueron: Revista TS Cuadernos Trabajo Social (Chile); Rumbos TS. Un espacio crítico para la reflexión en Ciencias Sociales (Chile); Cuadernos de Trabajo Social (España); Trabajo Social hoy (España); Alternativas. Cuadernos de Trabajo Social (España); Trabajo Social Global: revista de investigaciones en intervención social (España), y Voces desde el Trabajo Social (Puerto Rico). 
De la misma forma, se establecieron criterios de exclusión, lo que permitió distinguir la pertinencia de los artículos, acorde con la temática desarrollada. Así, se descartaron de la muestra aquellos estudios que, cumpliendo los criterios de inclusión, provenían de disciplinas distintas al Trabajo Social. Tampoco se consideraron revisiones sistemáticas, reseñas bibliográficas, entrevistas, presentaciones, cartas y/o declaraciones. Asimismo se excluyeron artículos en los que sus autorías $^{3}$ no contaban con al menos una persona con formación en Trabajo Social ${ }^{4}$.

El proceso de selección de estudios contó con tres fases: a) Fase 1: título y abstract. En esta etapa se revisaron los resultados arrojados en la indagación inicial, estableciendo, a través de la lectura de título y abstract, si cumplían o no los criterios de inclusión definidos; b) Fase 2: texto completo. Se revisó el contenido completo de todos los resultados seleccionados en la fase 1 para determinar si cumplían los criterios de inclusión/exclusión. En esta fase, y en virtud de la lectura, se consideró relevante incorporar nuevos criterios de exclusión, eliminando aquellos artículos que no dieran cuenta de la metodología utilizada para su realización. Asimismo, se excluyeron artículos que, teniendo alguna de las palabras clave definidas, y a juicio de las investigadoras, no contenían enfoque de género. Sumado a ello se eliminaron los artículos que, cumpliendo con los criterios de inclusión y exclusión señalados, no se situaban desde, en o para el Trabajo Social; c) Fase 3: evaluación crítica. Durante esta etapa, los estudios que pasaron la fase 2 fueron evaluados en su texto completo, estableciendo si cumplían o no con los criterios de inclusión y exclusión determinados.

El proceso de selección de estudios se desarrolló con un alto grado de reflexión, generando una discusión constante, para determinar el material con el que finalmente se trabajaría. En la primera fase se encontraron 52 artículos, con los que inicialmente se trabajó; en la fase 3, este número se redujo a 29 estudios, los que finalmente componen las unidades de análisis de la investigación desarrollada.

3 Respecto de las autorías, se excluyeron los artículos publicados por quienes suscriben este escrito.

4 Para ello se consideró la amplia gama de formaciones y titulaciones en servicio o trabajo social. 
Finalmente, se desarrolló un archivo Excel en el que se vertió la extracción de datos, que incluyó los siguientes ítems (tabla 2):

Tabla 2:

Extracción de datos

\begin{tabular}{ll}
\hline Información bibliográfica & $\begin{array}{l}\text { Autoría, nacionalidad de autores/as, } \\
\text { formación autores/as, año de publicación, } \\
\text { revista. }\end{array}$ \\
\hline Tipo de estudio & $\begin{array}{l}\text { Discusión teórica, investigación social, } \\
\text { sistematizaciones. }\end{array}$ \\
\hline Objetivos & Objetivos declarados. \\
\hline $\begin{array}{l}\text { Tipo de metodología } \\
\text { utilizada }\end{array}$ & $\begin{array}{l}\text { En los casos que sea declarada, se extrae } \\
\text { los principales aspectos metodológicos del } \\
\text { estudio. }\end{array}$ \\
\hline Características del estudio & $\begin{array}{l}\text { Enfoque teórico, ámbito territorial, sujetos } \\
\text { de estudio. }\end{array}$ \\
\hline Conclusiones & Síntesis de las conclusiones del estudio. \\
\hline
\end{tabular}

Fuente: Elaboración propia.

Los estudios, en su texto completo, fueron interpretados mediante la utilización de análisis de contenido el cual, según Capitolina Díaz y Pablo Navarro (1998) es un "conjunto de procedimientos que tienen como objetivo la producción de un meta-texto analítico en el que se representa el corpus textual de manera transformada" (p. 181). Flory Fernández (2002) destaca la idoneidad del análisis de contenido en las revisiones e investigaciones documentales; en este sentido, es certera en indicar que, en la actualidad, "en vez de faltar información sobra información" sobre diversos temas investigativos, "por lo que el problema estriba no tanto en conseguirla, sino en cómo digerirla adecuadamente" (p. 43). Tomando ambos elementos, se planteó un análisis general de los artículos científicos seleccionados por medio de tramas cualitativas generadas a partir de la propuesta de Díaz y Navarro (1998), quienes establecen estrategias extensivas e intertextuales. Las primeras se centran en la selección de algunos datos "a cambio de un tratamiento exhaustivo, completo y preciso de los elementos escogidos para el análisis" (p. 188); al mismo tiempo, en las estrategias intertextuales que se basan en la agrupación de datos en "distintos dominios analíticos y poder realizar comparaciones entre ellos” (p.189). Esta ta- 
rea fue asistida por el software de análisis de datos cualitativos Atlas. ti, versión 8.4, permitiendo un mejor almacenamiento, organización y sistematización de la información.

\section{Resultados}

\section{Decisiones metodológicas}

La revisión sistematizada nos permitió observar enclaves y posibilidades metodológicas que clarifican los caminos y alternativas posibles para indagar y estudiar fenómenos sociales. Por tanto, analizamos dichas decisiones metodológicas en las direcciones más ascendentes, exponiendo también el uso de técnicas, criterios éticos, análisis de información, entre otros, intentando abrir espacios hacia nuevas propuestas metodológicas desde y para la disciplina del Trabajo Social.

En este campo se observa un marcado acento hacia la metodología cualitativa, reconociendo sus valores intrínsecos, su carácter flexible, inductivo y subjetivo. La mirada microsocial que otorga al ejercicio indagativo permite un acercamiento comprensivo a los contextos y/o sujetos/as sociales que investiga, mediante el uso de diversas técnicas de recolección de datos como la observación, entrevista, conversación y documentos; sin embargo, se identifica a la entrevista (estructurada, semiestructurada y en profundidad) como técnica fundante para la recopilación de este tipo de información. Igualmente, hemos de indicar que los tres diseños de investigación más utilizados parten del estudio de caso, para analizar principalmente grupos de mujeres que comparten características comunes como nacionalidades, pertenencia étnica y de clase, edades, entre otras, en un contexto geográfico, sociohistórico y temporal específico. Le secunda el diseño fenomenológico, "tratando de averiguar el significado subjetivo que las informantes conferían a aquellas cuestiones sobre las que estaban siendo preguntadas" (Álvarez-Bernardo, 2017, p.16). Y, por último, el diseño narrativo, apuntando a que este permite la construcción de significados a partir de elementos discursivos y experienciales. Las modalidades de muestreo más frecuentes en las investigaciones estudiadas fueron el muestreo por conveniencia, ya que por razones prácticas se busca recopilar información en el menor tiempo posible, y el muestreo por bola de nieve, "para acceder a informantes clave que fueran surgiendo en las 
entrevistas" (Agrela, Romero y Fernández, 2017, p. 54). El apartado de análisis de datos es descrito escasamente, mencionando únicamente sus modalidades por medio del análisis de contenido o análisis de discurso. Se debe destacar que, en la gran mayoría de los artículos, no se menciona ni explica el procesamiento, organización y análisis de los grandes volúmenes de información considerados. Se destaca, además, el uso de análisis cualitativo asistido por computadora [CAQDAS] tales como Atlas.ti y NVivo.

En los artículos revisados se otorga un profundo realce a los principios éticos que sostienen la investigación social. Lo anterior puede estar ligado con la formación y ejercicio profesional del Trabajo Social y la importancia que desde ahí se entrega a los aspectos éticos de la tarea investigativa. De esta forma, las investigaciones mencionan y destacan resguardos de la privacidad, confidencialidad y el uso de consentimiento informado, además, realzan el derecho a desvincularse en cualquier etapa de la investigación, asegurando el anonimato, negociación y devolución de los resultados a publicar.

\section{El trazado de la teoría de género y sus viejos/nuevos temas}

La construcción y discusión teórica-conceptual de género acude a los aportes de autoras como Simone de Beauvoir (1949/1986), Kate Millett (1970/1995), Gayle Rubin (1975 y 1989), Carol Pateman (1995), Celia Amorós (1985 y 1994), Marta Lamas (1986; 1995 y 2000) y Marcela Lagarde (1990; 1996; 2001 y 2012), las que denominaremos como "pensadoras-madres" de la teoría de género y/o del pensamiento feminista.

Desde las "pensadoras-madres" se posiciona al género como una categoría analítica basada en una construcción social, cultural e histórica de lo implica ser mujer en una sociedad y temporalidad específica. Por lo que el dictamen de Simone de Beauvoir (1949) "no se hace mujer; se llega a serlo" (p. 371) es la base reflexiva que permite comprender que "el sexo es una categoría social impregnada de política" (Millett, 1970, p. 68), asumiendo que la "división de los sexos [es] socialmente impuesta. Es un producto de las relaciones sociales de sexualidad" (Rubin, 1989, p. 114). Lo anterior genera "un imaginario social con una eficacia simbólica contundente y, al dar lugar a concepciones sociales y culturales sobre la masculinidad y feminidad, es usado para justificar la discriminación por sexo (sexismo) y por 
prácticas sexuales (homofobia)" (Lamas, 2000, p. 4). Sin embargo, al ser un imaginario social, dichas relaciones de poder son posibles de transformar a partir de una "resignificación de la historia, la sociedad, la cultura y la política desde las mujeres y con las mujeres" (Lagarde, 1996, p. 13), por lo que la categoría género se transforma en "una herramienta esencial para develar que la asimetría presente en lo cotidiano puede ser interpelada, y que aquellas prácticas (...) [sexistas y homofóbicas] que se resisten a desaparecer, deben erradicarse" (González, 2019, p. 98).

A partir de este posicionamiento teórico-conceptual, la problematización en las investigaciones se realiza con base en las discusiones y postulados de organismos internacionales y sus respectivas normativas. Destaca el uso de datos provenientes de la Organización de Naciones Unidas para la Igualdad de Género y el Empoderamiento de la Mujer [ONU Mujeres], y las citas que refieren los acuerdos emanados de las conferencias mundiales sobre la mujer en Ciudad de México (1975), Copenhague (1980), Nairobi (1985) y, en especial, la Declaración y Plataforma de Acción de la Conferencia de Beijing (1995). En dichas instancias se instala una agenda de género y se proponen objetivos estratégicos a nivel mundial (destacando el Objetivo Estratégico del Desarrollo Sostenible para el año 2030 "lograr la igualdad entre los géneros y empoderar a todas las mujeres y niñas"), y se trazan líneas de acción sobre la situación y condición de las mujeres en los territorios donde están ubicados los estudios analizados. En tal contexto, es pertinente lo que plantea María Soledad Ascencio (2017) respecto de dejar de cuestionar la necesidad de incorporar el género a la agenda pública, sino más bien preguntar desde qué dispositivos ideológicos se está comprendiendo dicha perspectiva, analizar sus orientaciones metodológicas y construcciones teórico-conceptuales, ya que todas ellas impactan en las legislaciones, políticas públicas y programas nacionales/locales, enmarcadas en la protección y aseguramiento de los derechos de las mujeres, sin desconocer en ellas las lógicas de poder e influencia global-local.

La violencia de género y los cuidados son las principales temáticas indagadas. Éstas comúnmente parten de un diagnóstico poblacional y geográfico, dependiendo de la ubicación del estudio, y posteriormente se examina bajo la - ya revisitada - mirada de organismos 
internacionales, generalmente de informes de ONU Mujeres (2014), Organización Mundial de la Salud (OMS, 2002), Comisión Económica para América Latina y el Caribe (CEPAL, 2007; 2009; 2012 y 2013), y la Organización para la Cooperación y el Desarrollo Económico (OCDE, 2012). Dichos fundamentos apuntan a reconocer la importancia de la igualdad de género para impulsar procesos de desarrollo económico, social y ambiental en Latinoamérica, con el objetivo de la formulación, seguimiento y monitoreo de políticas de género, de carácter multiescalar y multifuncional, que apunten al abordaje, intervención y acción de los temas mencionados.

La violencia de género es descrita como un fenómeno sociocultural producto de la "estructura patriarcal que históricamente ha imperado en todas las sociedades y la asignación de roles en función del género" (Rodríguez, 2014, p. 74), fundamentada en la creencia de que el hombre es superior a la mujer (Flores, 2015), por tanto sus manifestaciones se basan en la discriminación, desigualdad y relaciones de poder entre los géneros (Moriana, 2015). Su expresión sigue siendo cotidiana y naturalizada, tal como menciona Daniela González (2019) en la restricción de las libertades como expresión del amor romántico en las relaciones de pololeo/noviazgo. Igualmente, posee un sinnúmero de expresiones (física, psicológica, sexual, económica, etc.), manifestaciones y niveles de agresión (Moriana, 2015). Los contextos geográficos, sociales e institucionales afectados/infectados continúan en aumento, cual pandemia mundial que nos afecta durante siglos - metáfora situada en los tiempos actuales de COVID-19- y que conlleva consecuencias directas en los cuerpos de las mujeres. En las sociedades impera desde la disminución de "habilidades intelectuales, pérdida de deseos o intereses, depresión, ansiedad y agotamiento psicológico" (Matulič-Domandzic, VicenteZueras, Boixadós-Porquet y Caïs-Fontanella, 2019, p. 59), hasta constituirse en un "obstáculo para conseguir la igualdad entre mujeres y hombres (...), diluyendo las oportunidades de las que debe disponer ésta con respecto a la igualdad jurídica, social, política y económica en la sociedad" (Fernández, 2015, p. 30).

A la par, revisamos los contextos sociales en los que se sitúan dichas violencias: procesos migratorios (Tudela, 2015; Bravo y Caparrós, 2015; Pavéz-Soto, 2016; Mut, 2017), relaciones de pareja (González, 
2019; Rodríguez y Ortiz, 2018), sectores rurales e indígenas (Zuñiga, 2014; Flores, 2014), desastres naturales (Cotarelo, 2015), sinhogarismo femenino (Matulič-Domandzic, Vicente-Zueras, Boixadós-Porquet y Caïs-Fontanella, 2019), encierro carcelario (De Miguel, 2014), centros de protección (Moriana, 2015), asociados al mercado laboral (Navarro y Aparicio, 2015) y su medicalización (Rodríguez, 2014).

Por su parte, los cuidados son indagados desde dos miradas. La primera se sitúa en la óptica de la economía feminista y economía del cuidado (Carrasco, 2009 y 2014; Pérez, 2014), observando que "la labor de las mujeres constituye una de las principales fuentes de acumulación capitalista, particularmente las labores de cuidado no remuneradas (...), que cumplirían un rol clave en el empobrecimiento sistemático de las mujeres" (Pérez y Troncoso, 2019, p. 14). Desde esta perspectiva, se instalan en el centro las desigualdades de género a partir de un análisis económico de las actividades y acciones cotidianas (productivas/ reproductivas) necesarias para la "sostenibilidad de la vida" (Ascencio, 2017). Le secunda el análisis desde el enfoque de derechos, considerando al cuidado como un derecho humano que reconoce que toda persona tiene derecho a cuidar, a ser cuidado y al autocuidado (Pautassi y Rico, 2001; Pautassi, Faur y Gherardi, 2004; Pautassi, 2007; Rico y Robles, 2016). Considera también que, en virtud de lo contemplado en el enfoque de derechos, es deber de los Estados diseñar e implementar políticas de cuidado de carácter universal, vinculantes tanto al mercado laboral (regulación de salarios, salud laboral, etc.) como "al interior de las familias y las comunidades", de tal forma que "se produzca y sostenga una redistribución de los tiempos de trabajo no remunerado, asumiendo la necesaria desfeminización de los trabajos de cuidados" (Ascencio, 2017, p. 190). Por tanto, estos enfoques teóricos apuntan a "problematizar al "sujeto productivo" del neoliberalismo, para politizar el cuidado y ubicarlo al centro de las relaciones humanas, partiendo de la base de nuestra mutua dependencia y la dimensión relacional y afectiva como intrínseca a las relaciones humanas" (Pérez y Troncoso, 2019, p. 17).

\section{Trabajo Social}

La historia del Trabajo Social está ligada a la construcción genérica de su identidad. Los estereotipos de género y, por ende, la socialización 
de género es un elemento fundamental en la comprensión del Trabajo Social (Rodríguez-Miñon, 2015). Desde sus inicios la profesión ha sido desarrollada casi exclusivamente por mujeres (Fernández-Montaño, 2015), quienes intervienen, mayoritariamente, con otras mujeres (Rodríguez-Miñón, 2016), de perfiles, características, sentires, corporalidades y realidades diversas (Mut, 2017).

A pesar de dicha constatación, se observan las dificultades que posee el Trabajo Social, como disciplina y profesión, en la incorporación del enfoque de género (Rodríguez-Miñón, 2016), a pesar de que su composición está marcada y caracterizada por la feminización de la profesión (Fernández-Montaño, 2015). Esta feminización está ligada a una desvalorización de sus saberes y prácticas, ligada a la posición social que tiene la disciplina en función del sexo y que es permanentemente afectada por la desigualdad de género (Rodríguez-Miñon, 2016). En el contexto patriarcal (Delgado, 2019), la feminización del Trabajo Social está necesariamente ligada a la idea de la feminización de los cuidados (Duarte, 2013), compartiendo con ellos una escasa valoración social (Rodríguez-Miñon, 2015).

Los cuidados son uno de los grandes objetivos de la intervención profesional del Trabajo Social (Fernández-Montaño, 2015). En relación con ellos, las autoras llaman la atención sobre el hecho de que los cuidados se organicen "exclusivamente en base al género" (Fuentes y Agrela, 2016, p. 48), excluyendo en muchos casos la función de las instituciones y el Estado en su ejercicio. Lo anterior implica una mirada hegemónica respecto de la idea de "familia" (Fuentes y Agrela, 2016), perpetuando las responsabilidades de las mujeres sobre la crianza e instaurándose un mandato moral asociado a la imagen de la "buena madre" (Agrela, Gutiérrez y Fernández, 2017). Este mirar hegemónico impide considerar las múltiples dificultades que las mujeres tienen a la hora de acceder a recursos y prestaciones (Mut, 2017), y, al mismo tiempo, perpetúa el modelo tradicional heterosexual (Álvarez, 2017).

\section{Un Trabajo Social desafiado}

En la literatura analizada es posible encontrar una serie de desafíos en torno a la relación entre Trabajo Social y la perspectiva de género. En ellos se encuentra la urgente necesidad de incorporar en la formación elementos asociados a la perspectiva de género (Rodríguez-Otero y 
Facal-Fondo, 2019), teniendo como marco los principios éticos de la profesión. No obstante, la perspectiva de género, por sí sola, no es suficiente para la comprensión de la realidad social; por ello, se requiere cruzarla con enfoques de diversidad cultural (Mut, 2017) y la incorporación de la interseccionalidad para cuestionar el sistema de presiones y categorías de poder (San Martín y Martínez, 2016; Mut, 2017; Delgado, 2019).

Así también, las autoras interpelan al Trabajo Social en varias dimensiones. Se menciona la necesidad de cuestionar los paradigmas a los que el Trabajo Social tradicionalmente acude (Mut, 2017), la prevalencia de la intervención individual por sobre la grupal y/o comunitaria (Tudela, 2015), los instrumentos utilizados en la intervención (Agrela, Gutiérrez y Fernández, 2017; Pérez y Troncoso, 2019), la rigidez de los modelos con los que se configura la comprensión de los entornos familiares (Mut, 2017; González, 2019), y la urgencia de problematizar y develar las estructuras de poder que subyacen a las relaciones sociales (Fernández-Montaño, 2015). En este sentido, la provocación está ligada a la redefinición de la práctica profesional, considerando que, para lograr una transformación social, es absolutamente necesario trabajar contra la opresión hacia las mujeres y contra toda forma de discriminación (Fernández-Montaño, 2015), resultando relevantes los cuestionamientos a las formas coloniales y occidentales inmersas en las intervenciones realizadas desde el Trabajo Social (Mut, 2017). Asimismo, en numerosos artículos se propone problematizar los supuestos utilizados por las profesionales en la intervención social, sobre todo respecto de las representaciones que las mismas realizan sobre las mujeres con quienes intervienen (Pérez y Troncoso, 2019), cuestionando la recurrencia de la idea de minorizar (Agrela, Gutiérrez y Fernández, 2017) y victimizar a las mujeres (Tudela, 2015; Pérez y Troncoso, 2019). De esta forma, se señala la necesidad de generar espacios en los que puedan ser trabajadas y cuestionadas las formas en que los mandatos de género se imponen y reproducen (González, 2019). Esto implica, a la vez, hacer conscientes cada uno de nuestros actos, reduciendo con ello, la posibilidad de generar y reproducir las estructuras opresivas (Fernández-Montaño, 2015), así como la oportunidad de develar las contradicciones y las formas de discriminación interiorizadas (Mut, 2017), y que son reco- 
nocibles en una serie de actitudes prejuiciosas y discriminatorias en el actuar profesional (Álvarez, 2017).

En respuesta a lo anterior, se releva la importancia de respetar procesos, puntos de vista y autonomía en la toma de decisiones de las mujeres y otredades (Mut, 2017; Tudela, 2015), incorporando las emociones y afectos en los modos en que se construye la identidad profesional (Agrela, Gutiérrez y Fernández, 2017). Asimismo, se menciona la necesidad de visibilizar "brechas, barreras e inequidades que enfrentan las mujeres en los diferentes espacios donde se desenvuelven" (Meyer, 2019, p. 89).

Por otro lado, resulta relevante la incorporación de la perspectiva de género en la formación (Delgado, 2019) e investigación realizada en Trabajo Social, visibilizando el sexismo y las formas patriarcales de interacción y comprensión de los fenómenos sociales (FernándezMontaño, 2015), lo que al mismo tiempo requiere incorporar las contradicciones generadas en el actuar profesional (Agrela, Gutiérrez y Fernández, 2017).

Otro de los elementos destacables es la existencia de dificultades para que las trabajadoras sociales ocupen cargos de poder, lo que es apreciado desde la formación, en la que existe un mayor número de hombres en cátedras y cargos de poder en las escuelas y facultades de Trabajo Social, y una sub representación de mujeres en la docencia, siendo aún menor que el número de estudiantes del mismo sexo (Díaz y González, 2019); a ello se suma una segregación horizontal y la existencia de un techo de cristal, en la que los hombres que ejercen el Trabajo Social tienen mayor presencia en "puestos con mayor contenido coactivo o de control social, y por otro, en puestos de dirección, control y gestión, ambos definidos socialmente como menos femeninos" (Rodríguez-Miñón, 2017, p. 37).

\section{Responsabilidad ético-política}

Elementos como la "feminización de las desigualdades" (Agrela, Gutiérrez y Fernández, 2017, p. 58), cuya mayor expresión se encuentra en la sobrerrepresentación de las mujeres en la franja de la pobreza (Mut, 2017; Agrela, Gutiérrez y Fernández, 2017), requieren enfrentar el tema de las desigualdades de género como un asunto de Derechos 
Humanos (Delgado, 2019) y, por ende, como un tema relevante en los posicionamientos que el Trabajo Social asume en su accionar ético y político.

En los textos revisados se aprecia un fuerte compromiso ético y político, asumiendo como eje la promoción de los derechos humanos y la justicia social (Fernández-Montaño, 2015), con especial atención a las diversas situaciones de desigualdad en las que viven las mujeres, teniendo claro que su origen se basa en el sistema patriarcal (FernándezMontaño, 2015), el cual afecta especialmente a quienes se encuentran situadas en los ejes de opresión (Delgado-Valentín, 2019). En este sentido, asumir una perspectiva de género en Trabajo Social implica revisar la forma en que se incluyen los derechos de las mujeres (Agrela, Gutiérrez y Fernández, 2017) en un trabajo comprometido en pro de su autonomía (Tudela-Vásquez, 2015; Mut, 2017; Agrela, Gutiérrez y Fernández, 2017) y empoderamiento (Zúñiga, 2014), la lucha contra toda discriminación (Graciet y Miranda, 2015), la visibilización de las otredades (Delgado-Valentín, 2019) y la problematización crítica de la forma en que la profesión y disciplina es responsable de la producción y perpetuación de las desigualdades de género (Agrela, Gutiérrez y Fernández, 2017).

La apuesta por la transformación social obliga a la profesión a revisar sus constructos y posiciones (Delgado-Valentín, 2019) y a repensar la ética en trabajo social desde la perspectiva de género, considerando elementos tan vitales como las emociones y contradicciones en el ejercicio profesional (Agrela, Gutiérrez y Fernández, 2017).

De esta forma, incorporar los temas apuntados permitiría asumir un proyecto ético-político profesional feminista, el cual implica una profunda "discusión crítica del modelo hegemónico patriarcal y la construcción de alternativas desde las otredades que permitan el desarrollo de estrategias que no reproduzcan la discriminación" (Delgado, 2019, p. 127). En este sentido, la creación de un proyecto ético profesional feminista en Trabajo Social requiere de un trabajo constante en torno a la modificación de las estructuras patriarcales y subvertir el orden masculino impuesto, generando cambios reales y concretos en la construcción genérica de la sociedad (Fernández-Montaño, 2015), cuyo primer reto sería la liberación de los constructos que limitan la posibilidad de un trabajo social emancipador (Delgado, 2019). 
La propuesta ético-política de un trabajo social feminista implica, a su vez, un emplazamiento político a cuestionar los roles de género y el ejercicio de los cuidados (Díaz y González, 2019), esto repercute en generar una exigencia y presión a los Estados (Rodríguez-Miñón, 2017) para que, a través de sus políticas públicas, puedan garantizar el pleno cumplimiento de los derechos de todas las personas (Matuli冈Domandzic, Vicente-Zueras, Boixadós-Porquet y Caïs-Fontanella, 2019). El trabajo social feminista interpela el proyecto ético-político del Trabajo Social, instalando la idea de que el proyecto emancipatorio requiere la "libertad de las otredades" (Delgado, 2019, p.129), y no solo su reconocimiento, cuestionando las configuraciones hegemónicas de los géneros, abriendo espacios para la diversidad y disidencia. En este sentido, la incorporación de "un análisis interseccional feminista como una guía inclusiva para la práctica profesional" (Delgado, 2019, p. 126) permitiría abrir la mirada con tal de observar la realidad social de una manera compleja, teniendo en cuenta los distintos ejes de opresión y haciendo frente a las múltiples desigualdades (Mut, 2017) desde una "posición situada, encarnada y subjetiva" (Agrela, Gutiérrez y Fernández, 2017, p. 62) que permita debatir, repensar y proponer un proyecto ético-político feminista y antipatriarcal.

\section{Discusión}

Las investigadoras sociales consideradas en esta revisión manifiestan, en más de una ocasión, que la perspectiva de género y/o feminista cruza transversalmente la investigación, empero existe una escasa vinculación entre las perspectivas teóricas y epistemológicas con el trazado metodológico, ya que se mantiene un apegado uso hacia las metodologías cualitativas y cuantitativas tradicionales, y se observa un "escaso riesgo" para indagar estas cuestiones con metodologías más atingentes. En esta revisión, una serie de autoras destacan la metodología feminista como "una orientación metodológica privilegiada para captar la forma en que las mujeres experimentan, contextualizan y significan el mundo" (Blázquez, Flores, y Berríos, 2012, p. 353). A la vez, validan y reconocen otros saberes, experiencias y conocimientos situados (Haraway, 1995) que, sin duda, "pueden nutrir prácticas de investigación e intervención menos sesgadas y discriminatorias" (Pérez y Troncoso, 2019, p. 21), produciendo conocimiento "desde la parcialidad, la loca- 
lización y la postura crítica, considerando, desde las posturas feministas, que es sólo desde ahí que tenemos la posibilidad de una búsqueda objetiva" (Galaz, Becerra, Álvarez y Hedrena, 2016, p. 58).

A la vez posicionan la técnica de producción narrativa como un "artefacto metodológico que opera promoviendo la localización de la mirada desde la cual se produce el conocimiento" (Balasch y Montenegro, 2003, p. 45), la que guarda un "doble propósito en la reformulación de las estructuras epistémicas, de modo de salvaguardar la agencia e intereses de las participantes, existiendo la intencionalidad de que las propias mujeres desde su voz construyan el objeto" (San Martín y Martínez, 2016, p. 42).

Por tanto, nuestra intención es generar una provocación metodológica en las investigaciones que realizamos desde el Trabajo Social en estas temáticas, que implique no solo trazar principios éticos, sino asumir un compromiso ético-político con las sujetas y contextos sociales que acompañamos/investigamos; en ello, definir nuestro lugar de enunciación y cuestionar los roles que se ponen en juego en el proceso investigativo. La provocación implica abrir puertas y ventanas que permitan airear la casa, para así dejar entrar ráfagas de renovadas ideas, propuestas y concepciones metodológicas que permitan observar el mundo desde la perspectiva feminista, que "implica asumir el desafío de visibilizar la condición de la mujer en [todas sus] dimensiones (...) en el marco de un sistema de relaciones sociales de género (desigual), con la pretensión de aportar a la transformación y la eliminación de las asimetrías" (Meyer, 2019, p. 76). En el mismo tenor, coincidimos en lo señalado por Teresa Kleba y Catarina Nascimento (2018), en relación a las posibilidades que entregan las epistemologías feministas para la comprensión de estos asuntos, proporcionando "pistas, caminos para repensar y resignificar los conceptos y las cuestiones sociales que involucran el hacer profesional del Trabajo Social”, y que, por tanto, la investigación feminista tiene la potencialidad de "proponer 'cuestiones de investigación' que se basan en la pluralidad, la diversidad y la multiplicidad de experiencias de las mujeres", utilizando "una combinación crítica de métodos de investigación y de perspectivas de análisis" (p. 32).

La construcción teórica desarrollada por las autoras se sitúa desde lo que hemos denominado las "pensadoras-madres" de la teoría de 
género y/o del pensamiento feminista, quienes, sin dudar, presentan una inconmensurable riqueza, tanto por la contribución al acervo teórico-conceptual, como en el abordaje activista y contestatario frente a los cánones androcéntricos de las ciencias sociales. Sin embargo, consideramos que los análisis siguen centrados en la noción de "género" desde una mirada totalizante capaz de explicarlo todo. Empero, las realidades sociales versan acerca de otra cuestión: actoras/es sociales que hablan/actúan desde sus propios cuerpos, forjando una inmensa diversidad de identidades, experiencias, saberes y relaciones interpersonales en el que están imbricadas otras categorías de opresión y desigualdad (raza/etnia, clase, orientación sexual, religión, otras), que esta mirada no es capaz de reponer. Además, si bien los datos que entregan organismos internacionales (ONU Mujeres, OCDE, CEPAL, entre otros) son pertinentes para contextualizar y muchas veces sustentar planteamientos e hipótesis investigativas, es necesario considerar al menos desde qué narrativas operan sus discursos globales-locales y, en ello, estar atentas al colonialismo discursivo (Mohanty, 2008/1991) y la violencia epistémica (Spivak, 2003/1985). Igualmente, se hace urgente referenciar el aporte que los movimientos feministas han realizado y siguen realizando con fuerza en los diversos escenarios, si bien sabemos que la teoría feminista está escrita en textos fundacionales, también está dada en el activismo, en el fuego de la acción y en la indignación callejera.

En esta misma línea nos tomamos de las palabras de Pérez y Troncoso (2019) al indicar que la mirada feminista nutre la perspectiva de género ya que permite "problematizar los supuestos discriminatorios que operan a la base (...) [de las investigaciones e intervenciones sociales,] reproduciendo a las mujeres como sujetos carentes, irracionales e ignorantes que deben ser" (p. 11) investigadas e intervenidas. Lo anterior es afirmado debido a que muchos de los estudios observados se han iniciado visualizando a las mujeres como víctimas (generalmente de violencia de género o de las cadenas globales/intergeneracionales de cuidados), carenciadas de habilidades y competencias para sobrellevar sus vidas, lo que conlleva a construir investigaciones e intervenciones sociales "sobre aquello que las mujeres no tienen y lo que no son" (p. 15). Lo anterior desconoce nuestras propias vulnerabilidades y la posibilidad de superarlas por medio de acciones de resistencia, 
pues se debe hacer presente que la vulnerabilidad es también "una fuerza movilizadora potencialmente efectiva en las movilizaciones políticas" (Butler, 2018, p. 28).

Necesitamos redirigir la mirada a este trabajo social desafiado, considerando para ello las palabras de Silvia Rivera Cusicanqui (2018), quien invita a "hacer comunidad sin silenciar las voces disidentes, las formas femeninas y ancestrales de crear lo político y crear el bien común" (p. 73). De esta forma, el reconocimiento de la corporalidad femenina, de los vínculos entre mujeres orientados a la reciprocidad y colaboración solidaria (Segato, 2016), permite reconocer y potenciar otras "formas de hacer política desde lo cotidiano/femenino", las que nos permitirían, desde una visión de comunidad tejido, "defendernos de las lógicas perversas del sistema capitalista" (Rivera Cusicanqui, p. 72). Esto nos lleva a reafirmar que la acción transformadora del Trabajo Social debe estar ligada a la actuancia y la responsabilidad éticapolítica feminista basada en la "micropolítica" que implica una acción desde los márgenes, una "política del cuerpo" situada en acciones cotidianas disidentes que convocan a "poner el cuerpo, hacerlo en un encuentro de comunidades de afectos, que quizás irradiarán hacia afuera y se conectarán con otras fuerzas e iniciativas, lejos de la competencia y de las estrategias del éxito" (p. 73), que permite sentipensarnos y tejer juntas rebeldías.

Por nuestra parte, postulamos que el Trabajo Social es una práctica de resistencia feminista, enmarcada en una política de los cuerpos, que permite establecer una "resistencia cotidiana" que "nutre así de repertorios a las multitudes (...) [por eso es urgente poner] atención a estas articulaciones de lo cotidiano con la política en el sentido más general" (Rivera Cusicanqui, 2018, pp. 69-70). De esta forma, comprender el Trabajo Social como una práctica de resistencia, es asumir una mirada crítica que desestabiliza el modelo basado en la reproducción de la igualdad y la injusticia, y cuestiona aquellas instituciones y prácticas que infligen violencia en las mujeres y disidencias (Butler, 2018, p. 40).

Estas cuestiones implican asumir un Trabajo Social en/desde la relación "territorio cuerpo-tierra" (Cabnal, 2010), ya que se posiciona en un espacio de lucha y resistencia situado en la pluralidad y diversidad de corporalidades y territorialidades que co-habitamos, en una "inter- 
conexión que hará brotar otro lenguaje de politicidad" (Rivera Cusicanqui, 2018, p. 73). Tomándonos de las palabras de Segato (2016), es un "proyecto histórico de los vínculos" ya que se compromete a "retejer y afirmar la comunidad y su arraigo vincular (...) buscar sus estrategias y estilo remontando el hilo de la memoria y los fragmentos de tecnologías de sociabilidad" (pp. 30-31), sostenido en una actuancia política femenina/feminista que "no es utópica sino tópica y cotidiana, del proceso y no del producto" (p. 31).

Es interesante señalar que los cánones androcéntricos de las ciencias sociales han calado profundamente en nuestra disciplina, en la producción del conocimiento y en la intervención profesional, por ello es necesario repensar las relaciones de subordinación disciplinar, los marcos interpretativos y la persistente mirada asistencialista y neoconservadora que aún ciñe nuestras mallas curriculares y permea nuestras intervenciones. Consideramos que, en estas discusiones, Gianinna Muñoz (2020) es certera en plantear la necesidad de "revisar la triada neoliberal-patriarcal-colonial en la discusión y en la formación disciplinar-profesional" (p. 8). En este sentido, reconocemos la posición de subalternidad en la que se ha situado al Trabajo Social en un mundo profundamente falocentrista y patriarcal, en el que se nos han realizado "marcaciones subalternizantes" por parte del "capitalismo moderno colonial" (Hermida, 2017, p. 136), entre las que se distingue el lugar que se nos ha atribuido, el tardío reconocimiento disciplinar, nuestra ubicación en el sur global y la precarización en la que gestamos nuestra labor. Sin embargo, "la posición de subalternidad de las intelectuales (mujeres) y del conocimiento femenino ha sido silenciada - paradójicamente- en las arenas postestructuralistas o postmarxistas" (Muñoz, 2020, p. 7), negando e invisibilizandonos de manera violenta. Una perspectiva crítica plantea una atención a las injusticias que genera la racionalidad neoliberal, considerando las diferencias de clase, etnia/raza, género y otras desde una mirada interseccional de las opresiones, considerando un "proyecto de sociedad que sea inclusivo, que goce con la diferencia y se escandalice con las desigualdades" (p. 9).

El Trabajo Social, como disciplina y profesión, tiene la responsabilidad ética y política de avanzar hacia prácticas interventivas no hegemónicas, que abran la posibilidad de construir espacios críticos de 
diálogo y problematicen, por ejemplo, la colonialidad del poder, dando cabida a una ecología de saberes, vivencias y sentires que exijan una imaginación epistemológica, que reconozca distintas prácticas y actores sociales (Sousa Santos, 2005), y que permita situarnos desde el sur global en torno a "epistemologías plebeyas" (Hermida, 2017, p.131), contrarrestando los efectos de un pensamiento tradicional que "produce ausencias y minoriza realidades" (p. 132). Sin embargo, la inclusión de epistemologías y posicionamientos disidentes implica, al mismo tiempo, generar y discutir propuestas formativas en las que se dote de valor a las pedagogías insurgentes y decoloniales, lo que implica repensar las categorías políticas y marcos analíticos en los que el Trabajo Social se mueve, desplaza y acciona, generando alianzas y aperturas con otras disciplinas y campos. Incorporar las epistemologías disidentes y abigarradas, aquellas que han logrado problematizar, cuestionar y tensionar el mundo académico y sus matrices patriarcales, heterocentradas y coloniales, es, a su vez, asumir el desafío de pluralizar los saberes y conocimientos relacionados con otras formas de habitar cuerpos y territorios desde el Sur. Lo anterior, en el reconocimiento de nuestra herencia e historicidad, nos enfrenta "al desafío de parir nuestra propia potencia plebeya disciplinar: la de una práctica social que pueda, desde sus propias subalternizaciones, ejercer la rebeldía y litigar para construir formas otras de pensar" (Hermida, 2017, p. 143).

Del mismo modo, la inclusión de un posicionamiento feminista implica el rechazo a todo tipo de jerarquización y exclusión, considerando las distintas posiciones que ocupan los grupos subalternos en nuestra sociedad. Lo anterior requiere, necesariamente, considerar las prácticas y narrativas sostenidas desde los territorios, como espacios de resistencia y re(ex)istencia desde lo local; al mismo tiempo, considera el valorar a las comunidades como sujetos políticos activos, potenciadores de propuestas de resistencia y subalternidad emancipadora.

\section{Conclusiones}

La revisión sistematizada permitió observar la constante labor que realizan las profesionales del Trabajo Social en forjar investigaciones e intervenciones sociales en esta presencia-ausencia, relación que no solo permite observar el mundo sino (con)vivir y (re)politizar la vida 
cotidiana. En ello constatamos la existencia de un Trabajo Social subversivo que piensa, actúa y siente desde los márgenes, donde muchas de estas acciones parecen invisibles, más hoy, en que todas unidas inspiran a seguir apostando por un Trabajo Social feminista emancipatorio y antipatriarcal.

Instamos a repensar las bases disciplinares tradicionales y cimentar este Trabajo Social desafiado, el que debe ser entendido no solo como un "proyecto" para seguir en el debate, sino más bien como un marco de acción que habilita a reflexionar y actuar con/para el Trabajo Social, (re)posicionar saberes femeninos/feministas, populares e indígenas, (re)situar los cuerpos y voces de actoras sociales en lucha y resistencia, defender lo comunitario y lo colectivo frente a lógicas individualistas y mezquinas. La construcción del conocimiento y el quehacer disciplinar implica una actuancia cotidiana, la que debe estar ligada a una responsabilidad ético-política acuerpada, que significa asumir una posición crítica-feminista.

Por consecuencia, debe existir una responsabilidad ético-política feminista que invite a forjar investigaciones e intervenciones "situadas", que cuestione las lógicas androcentristas y las relaciones de poder y dominación, explicitando nuestro lugar de enunciación, la inevitable implicación emocional y la urgente transformación social. Por ello, no basta con enunciar principios éticos y/o pilares del Trabajo Social, sino que es necesario contribuir a la transformación social real de la situación y condición de/con las mujeres en los territorios que compartimos.

Se requiere de la incorporación de las metodologías críticas feministas, que nos inviten a producir otros conocimientos, que continúen rompiendo los cánones androcentristas de la investigación desde la etnografía feminista, producciones narrativas, historiografía feminista y otras. Esto implica tanto la necesaria formación y capacitación, como la urgencia de compartir experiencias y saberes en la materia.

Es necesario trascender de la perspectiva de género al enfoque interseccional, ecología política feminista, economía feminista, ecofeminismo, feminismo decolonial, latinoamericano, comunitario y otros, que promueven una gama de conocimientos teóricos, saberes femeninos/feministas y prácticas políticas, y son capaces de observar las coyunturas/problemáticas sociales de las mujeres en los territorios 
que co-habitan, desde la pluralidad de sus corporalidades y territorialidades diversas y heterogéneas, advirtiendo que dicha comprensión/ acción promueve el desequilibrio y desmoronamiento efectivo de los dispositivos de poder y dominación que mantiene el statu quo de nuestras culturas políticas.

Finalmente, consideramos que el Trabajo Social feminista ha estado presente desde los inicios de nuestra disciplina, solapadamente se ha mantenido y resistido a los cánones androcéntricos de las ciencias sociales, al menosprecio de sus características feminizadas y su centramiento en el cuidado. Hoy reivindicamos esta presencia-ausencia y ello se cimienta en bases decoloniales, antirracistas, antipatriarcales y anticapitalistas. Tarea en extremo difícil, ya que no hay característica que zozobre ni sea redundante a su haber, en especial en la urgencia del actual contexto sociopolítico.

Todo lo anterior implica devolvernos al escenario real y vívido en el cual se desenvuelve el Trabajo Social, en la calle, en la militancia junto con movimientos y actoras/es sociales, en resistencia a la "nueva normalidad" y en lucha frente a las injusticias y opresiones interseccionales. Ese es el lugar donde debemos y tenemos que estar.

\section{Bibliografía}

Agrela, B. Gutiérrez, C. y Fernández, T. (2017). Repensar la ética en Trabajo Social desde una perspectiva de género. Cuadernos de Trabajo Social, 30(1), 51-64.

Álvarez-Bernardo, G. (2018). La maternidad en parejas de mujeres: reformulando el parentesco heteronormativo y biologicista. Alternativas. Cuadernos de Trabajo Social, O(24), 9-28. DOI: https://doi.org/10.14198/ALTERN2017.24.01

Amorós, C. (1985). Hacia una crítica de la razón patriarcal. Barcelona: Anthropos.

Amorós, C. (1994). Feminismo: igualdad y diferencia. México: UNAM.

Ascencio, M. (2017). Regímenes de bienestar y políticas de género en América Latina. Una nueva relación entre Estado, mercado, comunidades y familias. Revista Rumbos TS. Un espacio crítico para la reflexión en ciencias sociales, (16), 179-192.

Beauvoir, S. (1949/1986). El segundo sexo: los hechos y los mitos. Buenos Aires: Siglo XX. 
Beltrán, G. y Óscar, A. (2005). Revisiones sistemáticas de la literatura. Revista Colombiana de Gastroenterología, 20(1), 60-69.

Blázquez, N., Flores, F., y Berríos, M. (2012). Investigación feminista: Epistemología, metodología y representaciones sociales (Colección Debate y Reflexión ed.). Ciudad de México: UNAM.

Booth, A., Papaionnou, D. \& Sutton, A. (2012). Systematic Approaches to a Successful Literature Review. London: Sage.

Brazo, L. y Caparrós, N. (2015). Café y palabras. Las voces de las mujeres que cruzan fronteras. Cuadernos de Trabajo Social, 28(2): 225-234.

Brito, S., Posada, M., Basualto, L., Navia, G. y Castro, N. (2019). Narrativas de estudiantes universitarias. Derechos y oportunidades en contextos de INequidad de género. TS Cuadernos de Trabajo Social, 18, 98-119.

Brito, S., Basualto, L. y Posada, M. (2019). Los paradigmas emergentes de la identidad femenina. Resignificando los feminismos en espacios universitarios. TS Cuadernos de Trabajo Social, 19, 62-83.

Butler, J. (2017). Cuerpos aliados y lucha política. Hacia una teoría performativa de la asamblea. Buenos Aires: Paidós.

Butler, J. (2018). Resistencias. Repensar la vulnerabilidad y repetición. México: Paradiso Editores.

Cabnal, L. (2010). Acercamiento a la construcción de la propuesta de pensamiento epistémico de las mujeres indígenas feministas comunitarias de Abya Yala, en Feminismos diversos: El feminismo comunitario (pp. 11-25). Madrid: ACSUR-Las Segovias.

Comisión Económica Para América Latina [CEPAL]. (2013). Capital social y reducción de la pobreza en América Latina y el Caribe: en busca de un nuevo paradigma. Santiago de Chile: Naciones Unidas.

Comisión Económica para América Latina. (2009). Políticas para la inserción laboral de mujeres y hombres del Estado Plurinacional de Bolivia. Santiago de Chile: Naciones Unidas.

Comisión Económica para América Latina. (2012). El Estado frente a la autonomía de las mujeres. Santiago de Chile: Naciones Unidas.

Comisión Económica para América Latina. (2013). Participación laboral femenina y bono de género en América Latina. Santiago de Chile: Naciones Unidas. 
Canet, E., Martínez-Martínez, L. y Masiá, R. (2019). Experiencia profesional en intervención social para la prevención de la violencia de género en la Educación Secundaria. Trabajo Social Hoy, (87), 7-20. DOI: 10.12960/TSH.2019.0007

Carrasco, C. (2009). Mujeres, Sostenibilidad y Deuda Social. Revista de Educación, número extraordinario, 169-191, Ministerio de Educación y Cultura, España.

Carrasco, C. (2014). Con voz propia: la economía feminista como apuesta teórica y política. Madrid: La Oveja Roja.

Codina, L. (2018). Revisiones bibliográficas sistematizadas: procedimientos generales y Framework para ciencias humanas y sociales. Barcelona: Máster Universitario en Comunicación Social, Departamento de Comunicación, Universitat Pompeu Fabra. Recuperado de https://repositori.upf.edu/bitstream/ handle/10230/34497/Codina_revisiones.pdf

Cotarelo, L. (2015). Vulnerabilidad de las mujeres frente a la violencia de género en contextos de desastres naturales en Latinoamérica y Caribe. Trabajo Social Hoy, (76), 7-34. DOI: 10.12960/ TSH.2015.0013.

De Miguel, E. (2014). El encierro carcelario. Impacto en las emociones y los cuerpos de las mujeres presas. Cuadernos de Trabajo Social, 27(2), 395-404.

Delgado-Valentín, R. (2019). Más allá del binario: Un análisis crítico sobre la intersexualidad desde la perspectiva del Trabajo Social Feminista. Voces desde el Trabajo Social, 7(1), 110-133. DOI: https://doi.org/ 10.31919/voces.v7il.77.

Díaz, C. y Navarro, P. (1998). Análisis de contenido, en Métodos y técnicas cualitativas de investigación en ciencias sociales. Segunda reimpresión. Madrid: Editorial Síntesis.

Díaz, Y. y González L. (2019). Análisis de género en la educación universitaria en Trabajo Social en España. Cuadernos de Trabajo Social, 32(2), 351-363.

Duarte, C. (2013). Procesos de construcción del Trabajo Social en Chile. De historia, feminización, feminismos y ciencias. Revista Eleuthera, 8, 253-270.

Esquivel-Santoveña, E. y Osio, M. A. (2019). Violencia de pareja en poblaciones indígenas en México: Una revisión de la literatura. Trabajo Social Hoy, (87), 41-56. DOI: 10.12960/ TSH.2019.0009 
Fernández, F. (2002). El análisis de contenido como ayuda metodológica para la investigación. Revista de Ciencias Sociales, 96(2), 35-53. Recuperado de https://www.revistacienciassociales. ucr.ac.cr/images/revistas/RCS96/03.pdf

Fernández-Montaño, P. (2015). Trabajo Social Feminista: Una revisión teórica para la redefinición práctica. Trabajo Social GlobalGlobal Social Work, 5(9), 24-39. DOI: 10.30827/tsg-gsw. v5i9.3299.

Flores, N. (2015). Discriminación de género en relación al acceso a justicia para mujeres rurales víctimas de violencia familiar. Revista Rumbos TS. Un espacio crítico para la reflexión en ciencias sociales, (11), 51-62. Recuperado a partir de http:// revistafacso.ucentral.cl/index.php/rumbos/article/view/84

Fuentes-Gutiérrez, V. y Agrela-Romero, B. (2016). Migración femenina de "larga duración" y acuerdos de cuidados. Una mirada desde el Trabajo Social en origen y destino. Trabajo Social Global-Global Social Work, 6(11), 31-53. DOI: 10.30827/tsggsw.v6i11.4568.

Hermida, M. E. (2017). Contribuciones desde una epistemología plebeya al Trabajo Social frente a la restauración neoliberal. RevIISE: Revista de Ciencias Sociales y Humanas, 9(9), 127-145.

Jiménez, B. A. (2017). La búsqueda del feminismo en tiempos modernos. Trabajo Social Hoy, 81,49-58. DOI: 10.12960/ TSH.2017.0010.

Lagarde, M. (1990) Los cautiverios de las mujeres: madresposas, monjas putas, presas y locas. Centro de investigaciones interdisciplinarias en Ciencias y Humanidades, Universidad Nacional Autónoma de México

Lagarde, M. (ed). (1996). Género y feminismo. Desarrollo humano y democracia. Cuadernos Inacabados, (25).

Lagarde, M. (2001). Memoria Claves Feministas para la negociación en el amor. Managua: Puntos de Encuentro.

Lagarde, M. (2012). El feminismo en mi vida: hitos, claves y utopias. Ciudad de México: Instituto de las Mujeres.

Lamas, M. (1986). La antropología feminista y la categoría 'género'. Nueva Antropología, VIII(30): 173-198.

Lamas, M. (1995). Usos, dificultades y posibilidades de la categoría género. La Ventana, 1(1): 9-61. 
Lamas, M. (2000). Diferencias de sexo, género y diferencia sexual. Cuicuilco Nueva época, 7(18), 1-24. Recuperado de https:// www.redalyc.org/pdf/351/35101807.pdf

Lisboa, T. y Nascimento de Oliveira, C. (2018). El saber surge de la práctica: por un Trabajo Social con perspectiva feminista / Knowledge is nourished by the practices: to a Social Work by Feminist Perspective. Revista Rumbos TS. Un espacio crítico para la reflexión en ciencias sociales, (18), 11-36. Recuperado de http://revistafacso.ucentral.cl/index.php/rumbos/article/view/4

Galaz, C., Becerra, M., Álvarez, C. y Hedrera, L. (2016). Intervención social con mujeres inmigradas en Chile: $¿$ asistencialismo y/o promoción social? Revista Rumbos TS. Un espacio crítico para la reflexión en ciencias sociales, (14), 55-75. Recuperado de http://revistafacso.ucentral.cl/index.php/rumbos/article/view/35

García, C., Morales, M. Méndez, A., Delgado, M., Vilchis, F. y López, S. (2015). Confiabilidad y validez de un instrumento que mide percepciones de sexualidad. Revista Rumbos TS. Un espacio crítico para la reflexión en ciencias sociales, (12), 87-93. Recuperado de http://revistafacso.ucentral.cl/index.php/rumbos/article/view/73

Grant, M. J. y Booth, A. (2009). Una tipología de revisiones: un análisis de 14 tipos de revisión y metodologías asociadas. Revista Diario de información y bibliotecas de salud, 26(2), 91-108.

Greciet, C. y Miranda, A. (2015). Mujer, madre y abuela, la fusión de los roles. Revista Rumbos TS. Un espacio crítico para la reflexión en ciencias sociales, (12), 48-68. Recuperado de http://revistafacso.ucentral.cl/index.php/rumbos/article/view/71

González, D. (2019). Prácticas de control en relaciones de convivencia adolescente desde la perspectiva de género. Revista Rumbos TS. Un espacio crítico para la reflexión en ciencias sociales, (19), 95-108. Recuperado de http://revistafacso.ucentral.cl/ index.php/rumbos/article/view/330

Luis-González, C. y Aguilera-Ávila, L. (2019). Múltiple discriminación: homosexualidad y vejez. Trabajo Social Global-Global Social Work, 9(16), 225-247. DOI: 10.30827/tsg-gsw.v9i16.9322.

López-Zamora, M. y Ortega-Expósito, T. (2019). Atención integral a mujeres drogodependientes embarazadas. Propuesta de 
protocolo de actuación sociosanitaria. Trabajo Social Global-Global Social Work, 9(16), 248-267. DOI: 10.30827/tsggsw.v9i16.9180.

Martínez-de-Oporto, P. (2019). Una cuestión histórica de género. La desigualdad educativa y el aprendizaje de la mujer en España. Trabajo Social Global-Global Social Work, 9(16), 202224. DOI: 10.30827/tsg-gsw.v9i16.9330.

Matulic-Domandzic, M., De Vicente-Zueras, I., Boixadós-Porquet, A. y Caïs-Fontanella, J. (2019). Las mujeres sin hogar: realidades ocultas de la exclusión residencial. Trabajo Social GlobalGlobal Social Work, 9(16), 49-68. DOI: 10.30827/tsg-gsw. v9i16.8198.

Meyer, L. (2019). Promoción de la autonomía económica de las mujeres: un desafío para la sociedad chilena / Promotion of the economic autonomy of women: a challenge for chilean society. Revista Rumbos TS. Un espacio crítico para la reflexión en ciencias sociales, (19), 73-94. Recuperado de http://revistafacso.ucentral.cl/index.php/rumbos/article/view/32

Millet, K. (1970/1995). Sexual Politics Nueva York, Doubleday. Textos seleccionados de la versión en español: Política Sexual Cátedra, Feminismos clásicos.

Mohanty, M (2008/1991). De vuelta a 'Bajo los ojos de Occidente': la solidaridad feminista a través de las luchas anticapitalistas, en Descolonizando el feminismo. Teorías y prácticas desde los márgenes (pp. 407-463). Madrid: Cátedra, Universitat de Valencia, Instituto de la Mujer.

Morales, T. Peralta, M. y Bravo, L. (2015). Uso del espacio comunitarios y creencias de género en una muestra de hombres que ejercen violencia conyugal en la provincia de Biobío, Chile. TS Cuadernos de Trabajo Social, (13), 1-15.

Moreno, B., Muñoz, M., Cuellar, J., Domancic, S. y Villanueva, J. (2018). Revisiones Sistemáticas: definición y nociones básicas. Revista clínica de periodoncia, implantología y rehabilitación oral, 11(3), 184-186. DOI: https://dx.doi.org/10.4067/S071901072018000300184.

Moriana, G. (2015). Barreras para escapar de la violencia de género: la mirada de las profesionales de los centros de protección de mujeres. Cuadernos de Trabajo Social, 28(1), 93-102. 
Mut, E. (2018). Las refugiadas políticas colombianas en España: aportes para la disciplina de Trabajo Social de los enfoques de género e intercultural. Cuadernos de Trabajo Social, 31(1), 59-68.

Naudon, P. (2016). Mujeres migrantes en Chile: significaciones sobre su rol de madre y la crianza de hijos. Revista Rumbos TS. Un espacio crítico para la reflexión en ciencias sociales, (14), 99112. Recuperado de http://revistafacso.ucentral.cl/index. $\mathrm{php} / \mathrm{rumbos} /$ article/view/37

Navarro, G. y Aparicio, I. (2015). Desigualdades de género, pobreza y mercado de trabajo en Sal. Trabajo Social Hoy, 76, 47-74. DOI: 10.12960/TSH.2015.0015.

Organización de Naciones Unidas para la Igualdad de Género y el Empoderamiento de la Mujer [ONU Mujeres]. (2014). Guía estratégica empoderamiento político de las mujeres: marco para una acción estratégica. América Latina y El Caribe, 2014- 2017.

Organización para la Cooperación y el Desarrollo Económico [OCDE]. (2012). Cerrando las brechas de género: es hora de actuar. México: Organización para la Cooperación y el Desarrollo Económico.

Organización Mundial para la Salud [OMS]. (2002). Informe Mundial sobre la violencia y la salud. Naciones Unidas.

Parada, A. y Labrín, S. (2017). Sujetas de la casa: Procesos de producción de subjetividades de mujeres propietarias de vivienda social, asociadas al programa fondo solidario de elección de vivienda en la comuna de Chillán. TS Cuadernos de Trabajo Social, (16), 41-59.

Pateman, C. (1995). El Contrato Sexual. México: Editorial Anthropos.

Pautassi, L. y Rico, M. (2001). Licencias para el cuidado infantil. Derecho de hijos, padres y madres. Desafios: Boletín de la infancia y adolescencia sobre el avance de los Objetivos de Desarrollo del Milenio, 12, 4-12.

Pautassi, L., Faur, E. y Gherardi, N. (2004). Legislación laboral en seis países latinoamericanos. Avances y omisiones para una mayor equidad. Serie Mujer y Desarrollo, 56, Santiago de Chile: CEPAL.

Pautassi, L. (2007). El cuidado como cuestión social desde un enfoque de derechos. Serie Mujer y Desarrollo, 87, Santiago de Chile: CEPAL. 
Pavez-Soto, I. (2016). Violencia sexual contra niñas migrantes en Chile: polivictimización, género y derechos / Sexual violence against migrant girls in Chile: poly-victimization, gender and rights. Revista Rumbos TS. Un espacio crítico para la reflexión en ciencias sociales, (14), 113-131. Recuperado de http://revistafacso.ucentral.cl/index.php/rumbos/article/view/38

Pérez-Roa, L. y Troncoso, L. (2019). Deudas, mujeres y programas sociales en sociedades financiarizadas: resituando la "vida económica" en la intervención social. Revista Rumbos TS. Un espacio crítico para la reflexión en ciencias sociales, (19), 11-25. Recuperado de http://revistafacso.ucentral.cl/index. $\mathrm{php} / \mathrm{rumbos} /$ article/view/326

Pérez, A. (2014). Subversión feminista de la economía. Aporte para un debate sobre el conflicto capital-trabajo. Madrid: Editorial Traficante de Sueños.

Piedra-Cristóbal, J., Rosa-Martín, J. y Muñoz-Domínguez, M. (2018). Intervención y prevención de la violencia de género: un acercamiento desde el Trabajo Social. Trabajo Social GlobalGlobal Social Work, 8(14), 195-216. DOI: 10.30827/tsg-gsw. v8i14.6595.

Rico, M. y Robles, C. (2016). Políticas de cuidado en América Latina. Forjando la igualdad, en Serie Asuntos de Género, 154. Santiago de Chile: CEPAL.

Rivera Cusicanqui, S. (2018): Un mundo chíxi es posible. Ensayos desde un presente en crisis. Editorial Tinta Limón. Buenos Aires.

Rivera-Díaz, M., Varas-Díaz, N., Coriano-Ortiz, D., Padilla, M., ReyesEstrada, M. y Serrano, N (2015). Ellos de la calle, nosotras de la casa: el discurso patriarcal y las experiencias de mujeres que viven con el VIH/SIDA en Puerto Rico. Cuadernos de Trabajo Social, 28(1), 83-92

Rodríguez-Gónzalez, A. M. (2017). Análisis sociolaboral, con perspectiva de género, de la inmigración en la ciudad de Vigo. Trabajo Social Hoy, 80, 7-22. DOI: 10.12960/TSH.2017.0001.

Rodríguez, N. (2014). La medicalización de la violencia de género. Trabajo Social Hoy, 71, 73-86. DOI: 10.12960/TSH.2014.0004.

Rodríguez-Fernández, R. y Ortiz-Aguilar, L. (2018). Violencia de pareja, apoyo social y conflicto en mujeres mexicanas. Trabajo Social Hoy, 83, 7-26. DOI: 10.12960/TSH.2018.0001. 
Rodríguez-Miñon, E. (2017). La mujer como profesional del Trabajo Social. Trabajo Social Hoy, 80, 23-44. DOI: 10.12960/ TSH.2017.0002.

Rodríguez-Otero, L. y Treviño-Martínez, L. (2016). Sexismo y actitudes hacia la homosexualidad, la bisexuaidad y la transexualidad en estudiantes de Trabajo Social mexicanos. Trabajo Social Global-Global Social Work, 6(11), 3-30. DOI: 10.30827/tsggsw.v6il1.5181.

Rodríguez-Otero, L. y Facal-Fondo, T. (2019). Imaginarios, mitos y actitudes frente a la bisexualidad en estudiantes de Trabajo Social. Trabajo Social Global-Global Social Work, 9(17), 20-40. DOI: 10.30827/tsg-gsw.v9i17.8951

Rojas, C. (2017). Familias: transformaciones históricas y propuestas para el abordaje del Trabajo Social desde la perspectiva de género. Revista Rumbos TS. Un espacio crítico para la reflexión en ciencias sociales, (16), 93-119. Recuperado de http://revistafacso.ucentral.cl/index.php/rumbos/article/ view/48

Rubin, G. (1975). The Traffic in Women: Notes on the 'Political Economy' of Sex, in R. Reiter (ed.), Toward an Anthropology of Women (pp. 157-210). Nueva York: Monthy Review Press [trad. cast. (1986) El tráfico de mujeres: Notas sobre la 'economía política' del sexo. Revista Nueva Antropología, $\operatorname{VIII(30),~95-145].~}$

Rubin, G. (1989). Reflexionando sobre el sexo: notas para una teoría radical de la sexualidad, en: C. S. Vance (Comp.), Placer y peligro. Explorando la sexualidad femenina (pp. 113-190). Madrid: Ed. Revolución.

Segato, R. (2016). La guerra contra las mujeres. Madrid: Traficante de Sueños.

Spivak, G. (2003/1985). ¿Puede hablar el subalterno? Revista Colombiana de Antropología, (39), 297-364.

Tudela-Vázquez, M. (2015). Voces Migrantes Frente a la Violencia de Género: Una Investigación para la Acción en San Francisco (EEUU). Alternativas. Cuadernos de Trabajo Social, o(22), 185-206. DOI: https://doi.org/10.14198/ALTERN2015.22.11.

Valenzuela, A. (2014). Masculinidades y Estudiantes de Trabajo Social / Students Masculinities and Social Work. Revista Rumbos TS. Un espacio crítico para la reflexión en ciencias sociales, 
(9), 37-44. Recuperado de http://revistafacso.ucentral.cl/index.php/rumbos/article/view/112

Zúñiga Vásquez, M. (2014). Empoderamiento de mujeres quechuas y aymaras en la ruta de atención de violencia familiar y sexual a partir de una experiencia investigación-acción-participante. Revista Rumbos TS. Un espacio crítico para la reflexión en ciencias sociales, (10), 150-165. Recuperado de http://revistafacso.ucentral.cl/index.php/rumbos/article/view/104. 